\title{
Review
}

Journal of Innate

Immunity
J Innate Immun 2013;5:153-162

DOI: $10.1159 / 000342427$
Received: May 1, 2012

Accepted after revision: July 12, 2012

Published online: September 18, 2012

\section{Systems Biology of Circadian-Immune Interactions}

\author{
P.D. Mavroudis ${ }^{a} \quad$ J.D. Scheff ${ }^{b} \quad$ S.E. Calvano ${ }^{c} \quad$ I.P. Androulakis ${ }^{a-c}$ \\ Departments of ${ }^{\mathrm{a}} \mathrm{Chemical}$ and Biochemical Engineering and ${ }^{\mathrm{b}}$ Biomedical Engineering, Rutgers University, \\ Piscataway, N.J., and ' Department of Surgery, UMDNJ-Robert Wood Johnson Medical School, \\ New Brunswick, N.J., USA
}

\section{Key Words}

Circadian reprogramming $\cdot$ Circadian rhythm .

Clock entrainment $\cdot$ Clock genes $\cdot$ Clock synchronization •

Immune response $\cdot$ Inflammatory response

\section{Abstract}

There is increasing evidence that the immune system is regulated by circadian rhythms. A wide range of immune parameters, such as the number of red blood cells and peripheral blood mononuclear cells as well as the level of critical immune mediators, such as cytokines, undergo daily fluctuations. Current experimental data indicate that circadian information reaches immune tissues mainly through diurnal patterns of autonomic and endocrine rhythms. In addition, immune factors such as cytokines can also influence the phase of the circadian clock, providing bidirectional flow of circadian information between the neuroendocrine and immune systems. This network of neuroendocrine-immune interactions consists of complexly integrated molecular feedback and feedforward loops that function in synchrony in order to optimize immune response. Chronic stress can disrupt this intrinsic orchestration, as several endocrine signals of chronically stressed patients present blunted rhythmic characteristics. Reprogramming of biological rhythms has recently gained much attention as a potent method to leverage homeostatic circadian controls to ultimately improve clinical outcomes. Elucidation of the intrinsic properties of such complex systems and optimization of intervention strategies require not only an accurate identification of the signaling pathways that mediate host responses, but also a system-level description and evaluation.

Copyright $\odot 2012$ S. Karger AG, Base

\section{Introduction}

In order to cope with environmental challenges and optimize biological fitness, organisms adopt rhythmic variations in their physiological functions. In mammals, this intrinsic timing system is organized in a hierarchical manner where a light-sensitive master pacemaker synchronizes a bodywide web of cell-autonomous and selfsustained subsidiary clocks that are present in nearly every tissue of the body. The focal point of this system, which is commonly referred to as the master clock, is located in the suprachiasmatic nuclei (SCN) of the anterior hypothalamus of the brain. SCN neurons translate the photic signal of daily cycles to chemical information by altering the expression of various genes. Although SCN

\section{KARGER \\ Fax +4161306 1234 \\ E-Mail karger@karger.ch}

www.karger.com (c) 2012 S. Karger AG, Basel

$1662-811 X / 13 / 0052-0153 \$ 38.00 / 0$

Accessible online at:

www.karger.com/jin
Dr. Ioannis P. Androulakis

Biomedical Engineering Department, Rutgers University 599 Taylor Road

Piscataway, NJ 08854 (USA)

Tel. +1 7324454500 , ext. 6212, E-Mail yannis@ rci.rutgers.edu 
neurons adopt oscillatory behavior even in in vitro conditions independent of any external cues, the exogenous input of light-dark information ensures its synchronization to a 24-hour period. Similar behavior is observed in peripheral clocks which, in the absence of an entrainer, oscillate freely while falling out of sync, yet are synchronized in vivo by periodic physiological cues. Therefore, even considering the interactions between the central and peripheral clocks in isolation requires a system-level approach in order to gain understanding of the internal properties of the network. Obtaining fundamental and useful knowledge about these systems is even more difficult when one considers that the physiological systems regulated by circadian clocks also have their own complex internal dynamics.

One reason that understanding circadian rhythms is important is because they are associated with disease. Environmental desynchronization either by external stressors (e.g. shift work and jet lag) or by other genetic disorders may lead to vulnerabilities to infection and disease both in humans and in rodents. Abolishing master clock rhythmicity in mice by surgical ablation of the SCN seriously alters the daily rhythms of corticosterone, disturbs the rest-activity cycles, and ultimately leads to accelerated tumor growth. Furthermore, the lipopolysaccharide (LPS)-induced inflammatory response is magnified in jet-lagged mice relative to control animals, further leading to hypothermia and death after a certain period of time [1]. On the other hand, disease itself can impact on circadian rhythmicity. In particular, current experimental data show that systemic inflammatory diseases are associated with blunted rhythmicity of numerous intrinsic signals. For instance, sepsis has been associated with loss of diurnal rhythms of leptin and cortisol [2], and circadian rhythmicity of cortisol has been shown to be prognostic of longer survival in patients with metastatic breast cancer [3].

The intersection between circadian rhythms and the inflammatory response, both governed by complex signaling networks, truly necessitates systems-biologybased investigation if we are to understand the relationships between these systems and leverage this knowledge towards practical ends. This requires both experimental and computational approaches aimed at understanding circadian rhythms, inflammation, and their interactions. In this review, we discuss knowledge acquired in recent years relative to the bidirectional links between circadian and immune responses and the occasions where their rhythmic orchestration is disrupted, as well as current knowledge relative to the reprogramming of endogenous rhythms. We present systems biology approaches that have been leveraged in order to gain insight into these networks.

\section{Mechanistic Insight into Circadian Entrainment of Central and Peripheral Clocks}

The core clock elements that give rise to circadian timekeeping in mammalian SCN and found in most, if not all peripheral cells, are a group of so-called clock genes. Mouse SCN involves three period genes (Perl, Per2, and Per3), two cryptochrome genes (Cryl and Cry2), two basic helix-loop-helix transcription factors (CLOCK and BMAL1), and two orphan nuclear hormone receptors (REV-ERB $\alpha$ and ROR $\alpha$ ). Negative and positive feedback interactions among these clock genes lead to transcriptional oscillations that retain an approximate 24-hour periodicity independent of any external entrainer. In order to stay in harmony with environmental changes, clock gene oscillations must be corrected on a daily basis by entraining signals. As such, after receiving the environmental input from light/dark cycles, SCN neurons deliver photic information to the periphery of the body via direct routes such as the circadian secretion of hormones and the neuronal activity of the autonomic nervous system, and indirect routes such as the daily rest-activity cycles that further control the feeding time. This network of interactions among SCN and peripheral tissues reveals a nested level of biological organization where circadian information is retained at various levels of mammalian physiology from rhythmic patterns of clock gene expression to behavioral rhythms of sleep/wake cycles. Systemic integrity is largely dependent on the coherent function of the sub-systems composing the network. Their circadian rhythmicity is further related to the integrity of humoral and neuronal entraining signals, further underscoring the need of a system-level approach in order understand the underlying properties and design principles.

Quantitative mathematical models have been applied to gain mechanistic insights into clock gene network function [4-9] (table 1). Among them, Becker-Weimann et al. [7], using a reduced model to simulate clock gene network, denoted that the negative feedback loop among Per and Cry genes is critically important for the maintenance of clock gene oscillations. They showed that even if the positive feedback loop among Bmal, Ror and Reverb $\alpha$ genes is substituted by a constantly expressed activator, oscillations can still occur. These modeling results were supported by the experimental evidence showing 
Table 1. Mathematical models relative to clock genes, entrainment of clock genes, and immune/clock dynamics in published studies

\begin{tabular}{|c|c|c|}
\hline Dynamics of interest & $\begin{array}{l}\text { Mathematical } \\
\text { formulation }\end{array}$ & Findings \\
\hline \multicolumn{3}{|l|}{ Clock gene dynamics } \\
\hline Leloup and Golbeter [4] & ODE & Autonomous oscillations with adverse phase of Per and Bmal1 mRNAs in the dark period \\
\hline Mirsky et al. [5] & ODE & Predicts phenotypes of 7 single- and 2 double-knockout mutations of clock genes \\
\hline Gallego et al. [8] & ODE & The casein kinase mutant $\left(\mathrm{CKI}_{\varepsilon}{ }^{\tau}\right)$ increases kinase activity \\
\hline Westermark et al. [9] & SDE & $\begin{array}{l}\text { Predicts that robust oscillations in peripheral cells found experimentally may be in reality } \\
\text { damped oscillations driven by noise }\end{array}$ \\
\hline
\end{tabular}

Entrainment of clock gene dynamics

Abraham et al. [12] ODE

\begin{tabular}{lll}
\hline Antle et al. [13] & ODE & \begin{tabular}{l} 
Rhythmic regulation in the SCN region involves arrhythmic (gate) and oscillatory cells \\
\hline Antle et al. [14]
\end{tabular} \\
\hline ODE & $\begin{array}{l}\text { Reveals that the previous model can be entrained by a circadian input and maintain phase } \\
\text { response curve similar to what was observed experimentally }\end{array}$ \\
\hline Geier et al. [15] & ODE & $\begin{array}{l}\text { The number of oscillators and their connectivity are important for synchronization } \\
\text { dynamics as well as their periodic behavior }\end{array}$ \\
\hline Gonze et al. [16] & ODE & $\begin{array}{l}\text { Importance of phase response curve shape for entrainment dynamics } \\
\text { the individual cell oscillations }\end{array}$ \\
\hline Mavroudis et al. [11] & SDE & $\begin{array}{l}\text { Cortisol synchronizes peripheral clock genes in an amplitude- and frequency-dependent } \\
\text { manner }\end{array}$ \\
\hline
\end{tabular}

\section{Immune/clock dynamics}

Meyer-Hermann ODE

et al. [28]

Scheff et al. [21] ODE

Scheff et al. [30] ODE Semi-mechanistic link between generation of heartbeats and immune compartments

ODE = Ordinary differential equations; SDE = stochastic differential equations.

that $R e v-e r b \alpha^{-1-}$ mutant mice have rhythmic behavior even though their positive feedback mechanism is not functional. More recently, Mirsky et al. [5] used a detailed model to predict the phenotype of 7 knockout and 2 double knockout mutations as well as concentration variations in clock genes in the respective scenarios. However, as it was noted in their work, in order to describe phenotypes observed at tissue or organ level, additional dynamics such as entrainment and synchronization must be taken into consideration.
Recently, the mechanism of peripheral entrainment by systemic cues has been further elucidated. Single-cell experiments of Nagoshi et al. [10] in rat and mouse fibroblasts showed that single peripheral cells even in in vitro conditions independent of any entraining cue retain robust rhythmicity similar to SCN cells. As a result, the dampening rhythms they observed in vitro at the population level emerge from robust single-cell oscillations that fall out of phase and desynchronize in the absence of an entraining force. Based on this, we recently studied the 
synchronization properties of cortisol in a population of peripheral clock genes. We found that cortisol entrains the peripheral clocks in an amplitude- and frequencydependent manner [11] (table 1). Chronically stressed conditions, represented by blunted cortisol circadian amplitudes, lead to loss of peripheral clock gene entrainment and synchronization. Similarly, cortisol oscillatory frequencies largely deviating from a 24-hour period, which have been experimentally found in rodent models of jet lag, fail to synchronize peripheral clocks resulting in a high level of desynchronization and weaker ensemble average rhythm. Additionally, there has been a lot of interest relative to entrainment of central and peripheral clocks, and several mathematical models have been constructed in order to characterize their underlying dynamics [12-17] (table 1). In particular, Abraham et al. [12] showed that the oscillator qualities that greatly determine entrainment efficiency are the ratio between entrainer coupling strength and oscillator amplitude, and the rigidity of the oscillatory system as defined by the relaxation rate upon perturbation (table 1). This result can explain the evidence that peripheral tissues such as the lung retain significantly different entrainment characteristics than the SCN since SCN neurons due to their intercellular coupling retain characteristics of rigid oscillatory dynamics.

Deciphering entrainment dynamics of peripheral cells is very important since several genes that are critically involved in the immune function are regulated by peripheral clock genes. These genes are commonly referred to as clock-controlled genes (CCGs). Transcription factors such as signal transducer and activator of transcription 3 and 5 (STAT3 and STAT5), as well as nuclear factor$\kappa \mathrm{B}(\mathrm{NF}-\kappa \mathrm{B})$ are directly regulated by the molecular clock and exhibit circadian rhythmicity in humans and rodents. Further, these transcription factors participate in cytokine signaling pathways and as such they indirectly regulate innate and adaptive immune responses. As a result, a robust circadian signal of clock genes implies the efficient delivery of circadian information to the immune response of the body. In addition, circadian information through metabolic, neuronal, and humoral entraining signals reaches organs of the immune system, such as lymph nodes and spleen. Outputs of this entrainment, among others, are the rhythmic variation of critical immune components such as natural killer cell (NK) levels and cytokine expression that ultimately control critical immune responses such as NK cytotoxicity, phagocytosis, and inflammatory responses [18]. On the other hand, the temporal variations in immune mediators tune the central clock by affecting critical behavioral rhythms, such as sleep/wake patterns, forming a feedback interaction between circadian and immune systems.

\section{Bidirectional Communication between Circadian Clock and Immune Systems}

The complexity inherent in both the inflammatory response and the hierarchical system of circadian clocks necessitates a systems biology view to study how these systems interact. One useful experimental technique to study systemic inflammation is the human endotoxemia model. Human endotoxemia consists of the administration of low doses of endotoxin (LPS) to healthy human volunteers. Through the binding of LPS to its Toll-like receptor 4 (TLR4), endotoxemia provokes physiological changes that, in part, mimic those occurring in acuteand chronic-inflammation-linked diseases such as sepsis and trauma. Thus, human endotoxemia functions as a practical model of TLR4-agonist-induced systemic inflammation by eliciting neuroendocrine, hemodynamic, and leukocyte transcriptional responses [19]. Furthermore, circadian properties of the endotoxemia response have been studied both experimentally [20] and computationally [21].

\section{Neuroendocrine and Autonomic Circadian Regulation of Immune Responses}

The master clock in the SCN governs the central release of circadian hormones and signals which travel throughout the body and entrain the peripheral oscillators to a consistent phase. Interestingly, as summarized in figure 1 , many of these circadian signal transduction mediators also play roles in regulating immune responses, such as cortisol, melatonin, and the autonomic nervous system.

One of the direct routes through which the central clock entrains peripheral tissues is by the production of glucocorticoids (cortisol, in humans) in the adrenal gland. In addition to being notable for its clear circadian pattern in homeostasis, cortisol is a key anti-inflammatory hormone. Balsalobre et al. [22] further showed that glucocorticoids can induce circadian gene transcription in rat-1 fibroblasts as well as phase shift clock gene expression in the liver, kidney, and heart without influencing clock gene expression in the central clock, as the glucocorticoid receptor is not expressed in the SCN [22]. In addition, experiments of Burioka et al. [23] illustrated that administration of dexamethasone, a synthetic gluco- 
corticoid, in human bronchial epithelium and peripheral blood mononuclear cells (PBMCs) in vitro and in vivo significantly influenced the expression of the PER 1 clock gene. Interactions between glucocorticoids and circadian rhythms have been studied through pharmacokinetic and pharmacodynamic modeling to understand the pharmacological implications of endogenous circadian rhythmicity. Glucocorticoids exert downstream effects through a signaling pathway that includes the binding of glucocorticoids to glucocorticoid receptor molecules, translocation of the activated receptor complex to the nucleus, and transcriptional regulation by binding to glucocorticoid-responsive elements in the promoter region of target genes. Experiments in mouse peripheral organs as well as mesenchymal stem cells indicated that Perl and Per2 clock genes contain glucocorticoid-responsive elements in their promoter regions and therefore are directly regulated by glucocorticoid signaling pathways. Since glucocorticoids interact with clock genes and regulate their expression, they indirectly modulate clock-mediated pathways and CCG (e.g. STAT3, STAT5, and NF-кB). This results in far-reaching effects, as glucocorticoids influence circadian cytokine production, leukocyte distribution, proliferation, and apoptosis.

The circadian secretion of melatonin may also regulate the expression of immune mediators such as cytokines. The primary mechanism of melatonin production is by the pineal gland of the brain, which is tightly regulated by the SCN. In addition to operating as a circadian-entraining hormone, melatonin conveys a significant immunomodulatory effect. For instance, the peak melatonin circadian rhythm at night has been correlated with the nocturnal rise in blood T lymphocytes. Pinealectomy, on the other hand, is followed by overall immunosuppression, likely mediated by the reduction in lymphocytes and other cytokines, such as interleukin (IL)-2, IL-12, and tumor necrosis factor (TNF)- $\alpha$, that naturally assist the host to mount humoral responses [24]. Furthermore, in murine bone marrow cells, administration of melatonin appears to induce immunity by inhibiting apoptosis during early B-cell development [25]. Melatonin plays also a role in the development and growth of cancer since its production is correlated both with a reduction in the IL-10 anti-inflammatory cytokine that has cancer-growth-promoting activity and with an increase in human monocytes to produce the IL- 6 cytokine that has cancer-stimulatory activity.

The autonomic nervous system, through both its sympathetic and parasympathetic efferent arms, also conveys circadian information to the immune system. Light/dark information reaches the autonomic system through in-

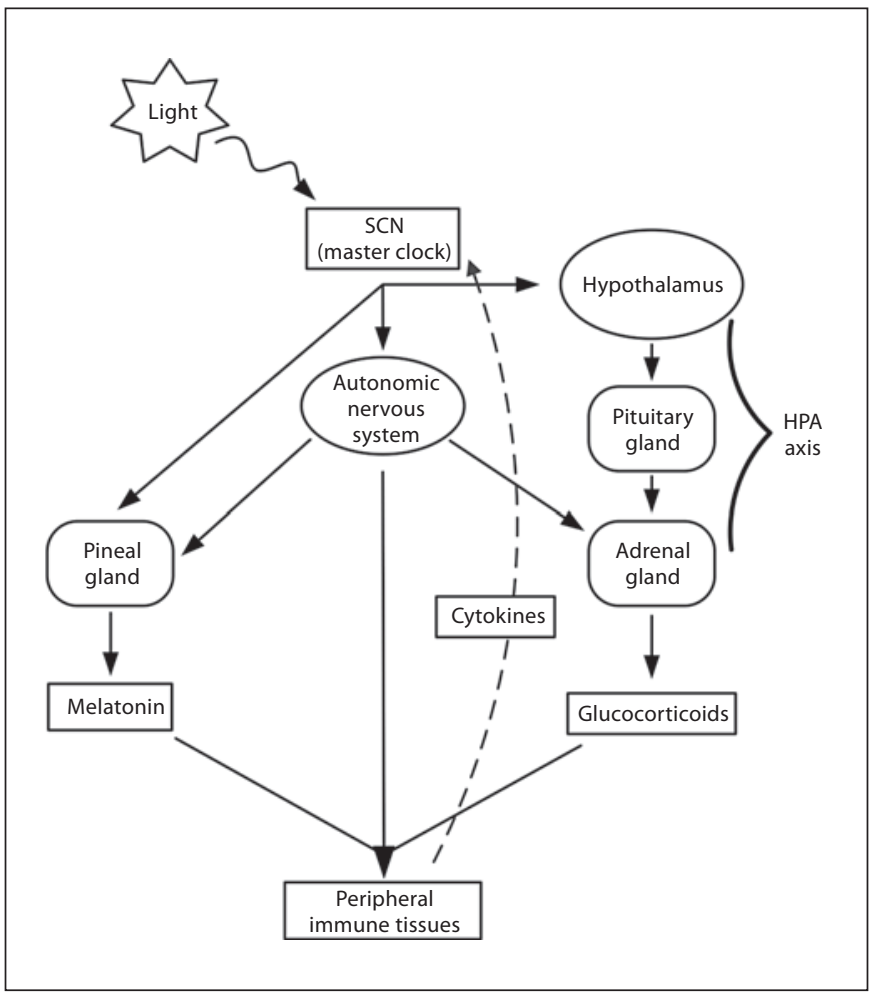

Fig. 1. Schematic review of some components of the bidirectional communication between circadian clocks and inflammatory mediators.

hibitory and excitatory inputs from the SCN to the paraventricular nucleus that controls pre-autonomic neurons and ultimately regulates sympathetic and parasympathetic activity. Autonomic activity is then mediated to the periphery of the body through autonomic innervation of various peripheral organs. The adrenal and pineal glands are innervated by autonomic projections and as such there is an indirect autonomic regulation of immunity embedded in the secretion of cortisol and melatonin, respectively. Additionally, primary and secondary lymphoid organs such as the spleen and liver receive extensive autonomic input. Upon stimulation, sympathetic nerve terminals in the spleen secrete norepinephrine that ultimately mediates activity of NK cells and macrophages. Recently, it has been shown that the norepinephrine input to the spleen retains diurnal rhythmicity, further illustrating the role of the autonomic nervous system as a conveyor of photic information [25]. Interestingly, in the same experiment, sympathetic denervation disrupted the diurnal variations of splenocyte cytokines and NK cells. Similarly, hepatic NK cells are also regulated by the 
circadian sympathetic input of the liver. The autonomic nervous system also mediates immunomodulatory effects by the cholinergic anti-inflammatory pathway through the release of acetylcholine from reticuloendothelial organs such as the spleen, liver, and heart that further interact with acetylcholine receptors on tissue macrophages and ultimately inhibit the release of TNF, IL-1, and other cytokines [26]. This, combined with the autonomic innervation of critical lymphoid and reticuloendothelial organs, allows for autonomic regulation of the inflammatory response.

These and other centrally mediated dual circadian and inflammatory signals impose a circadian character on inflammatory responses. Blood stimulated ex vivo with LPS at different times throughout the circadian cycle results in significant circadian rhythms in the peak responsiveness of cytokines. In vivo human endotoxemia experiments showed that there is a significantly larger increase in cortisol levels as well as in body temperature when LPS is injected into healthy volunteers in the evening (when cortisol levels are low) versus in the morning (when cortisol levels are high) [27].

Meyer-Herman et al. [28] developed a mathematical model to evaluate the neuroendocrine-immune system interactions specifically in the context of rheumatoid arthritis (table 1). This model describes mainly the measured circadian responses of plasma levels of TNF, noradrenaline, and cortisol, making use of a set of ordinary differential equations. The model was calibrated with experimental data of healthy subjects and rheumatoid arthritis patients. Importantly, they predicted through mathematical modeling that treatment with glucocorticoids between 00:00 and 02:00 a.m. induced the strongest inhibitory effect on TNF secretion. In chronic inflammatory diseases such as rheumatoid arthritis where patients are characterized by an overexpression of inflammatory responses, to reduce pro-inflammatory mediators such as TNF is often a clinical target. Similarly, Scheff et al. [21] incorporated a multilevel mathematical modeling scope based on which they evaluated the interplay between inflammation and circadian rhythms (table 1). This model predicted that LPS administration during the night induces larger increases in inflammatory mediators and larger reductions in the heart rate variability (HRV) relative to administration in the morning. HRV, defined as the distribution of time intervals between successive heartbeats, is considered as a potential prognostic marker in systemic inflammatory diseases such as sepsis, with lower values being generally associated with poorer health outcomes. Relative to this, the modeling results of
Scheff et al. [30] lie in accordance with experimental data showing that septic patients have a significantly increased risk of mortality at night [29]. Extension of this model, further incorporating a mathematical description of the sympathovagal signals that give rise to heartbeats and ultimately to HRV [30] (table 1), allowed for further investigation of the mechanistic underpinnings of the inflammatory responses that ultimately lead to changes in HRV. The importance of this model lies in the fact that it incorporates a semi-mechanistic-based representation of circadian heart rate dynamics that includes their derivation from cellular, molecular, and neural signals enabling the evaluation of multiple in silico scenarios relative to physiology underpinning changes in HRV.

\section{Immune Mediators Regulate the Circadian Clock}

Just as the mechanisms described above convey circadian information to the immune system, mediators produced in the inflammatory response can in turn modulate the function of the circadian clock. It is well established in vitro that LPS-induced responses can exert significant effects on the circadian clock mechanism. Administration of LPS to SCN slices increases the secretion of arginine-vasopressin neuropeptide. Originally, the rhythmic expression of arginine-vasopressin is directly regulated by SCN [31]. This suggests that the neuroendocrine output of SCN can also be modified by immune challenge and ultimately influence the behavioral rhythms of the host. In accordance with this, Marpegan et al. [32] showed that intraperitoneal injection of LPS in mice induced phase delays to their locomotor activity. These phase shifts to rodent activity were present only if LPS was administered specific times of the day, illustrating a time of day dependency of inflammatory outcome. As it can be implied by the experiment of Marpegan et al. [32], inflammatory stimuli even at the periphery of the body can trigger alterations in the circadian clock of the organism. In particular, Okada et al. [33] showed that intravenous injection of LPS to rats results in significant suppression of the clock gene Per2 and the CCG $D b p$ in the SCN underlining a direct effect of peripheral inflammatory stimuli on the central circadian clock of the body.

It is likely that the precise mechanism through which LPS mediates its downstream effects on the SCN and peripheral tissues involves cytokines which are released in response to inflammatory stimuli (fig. 1). Relative to the interactions of cytokines and the central clock, Kwak et al. [34] found that long-term treatment of rat SCN cultures with interferon (IFN)- $\gamma$ blunts the diurnal rhyth- 
micity of Perl even at the level of single cells. Similarly, a cocktail of TNF- $\alpha$, LPS, and IFN- $\gamma$ caused a decrease in the SCN neuronal-firing rhythmicity. Beynon and Coogan [35] further showed that in rodent SCN, the IL$1 \beta$ pro-inflammatory cytokine receptor IL-1R1 is rhythmically expressed. In addition, a peripheral immune challenge by a large dose of LPS significantly upregulated IL-1R1 along with critical components of the IL- $1 \beta$ signaling pathway, such as c-Fos and p65-NF- $\kappa$ B. This suggests that the central clock is directly sensitive to immune challenges from the peripheral tissue. Numerous experiments have shown that the brain receives inflammatory signals from the periphery of the body in response to injury/infection. This signaling has been implicated in the exacerbation of sickness, development of symptoms like depression, and in the impairment of numerous diurnal rhythms such as temperature and melatonin.

As it is the case for LPS, cytokines cause also alterations in the peripheral clock function. Treatment of human hepatocytes with IFN- $\alpha$ induces downregulation in the expression of CLOCK and BMAL1 genes in a STAT1 transcription factor-dependent manner [36]. Furthermore, through both in vitro and in vivo studies, Cavadini et al. [37] showed that TNF- $\alpha$ and IL-1 $\beta$ downregulated the expression of mouse Per1-Per3, and Dbp, clearly illustrating that the output of the clock network is also regulated by cytokine signaling. However, our current mechanistic understanding of these interactions is not sufficient to explain the temporal dynamics observed in endotoxemia experiments, suggesting that there are likely more undiscovered links between circadian rhythms and inflammatory responses [20].

The extent to which LPS injection modulates the central clock in vivo in humans is still a topic of debate. An endotoxemia study done in humans [20] found that melatonin levels, a proxy for the function of the circadian clock, did not change in response to endotoxemia. However, unlike the rat endotoxemia study which directly analyzed gene expression in the SCN, transcriptional analysis was only done in peripheral blood leukocytes, which exhibited similar regulation of clock genes. Thus, it could be that like the effect of feeding on the central clock but unlike the effect of light, endotoxemia primarily effects peripheral clock function. Further studies, including injections at more time points, will be required to further investigate the issue of central clock regulation by LPS, but it is clear that peripheral circadian clocks can be significantly perturbed in endotoxemia. This could indicate a loss of coupling between the central and peripheral clocks under stress.

\section{Disruption of Circadian Rhythmicity of the Body - Reprogramming of Biological Rhythms}

There is a fair amount of evidence indicating that several chronically stressed conditions are correlated with disruption of biological rhythms such as sleep/wake cycles, circadian rhythms of immune mediators and diurnal oscillations of hormones [3]. Chrousos and Gold [38] further showed that a chronically stressed hypothalamicpituitary-adrenal axis is characterized by a decreased variance in cortisol both due to evening nadir elevation and to morning zenith decreases. In critical illness, this has been hypothesized to be driven by elevated levels of inflammatory mediators, as well as neural input to the adrenal, directly stimulating glucocorticoid secretion. Circadian rhythms in melatonin were observed to be suppressed in septic patients indicating a loss of central clock rhythmicity due to the tight link between SCN function and melatonin secretion [39]. However, non-septic patients in the intensive care unit still had circadian rhythms in melatonin suggesting that there may be subtle diseasespecific mechanisms driving the specific characteristics of the loss of circadian rhythmicity in critical illness.

Disruption of circadian rhythmicity may also be presented as a phase delay or advance in diurnal rhythmicity. In particular, Alesci et al. [40] showed that patients suffering from the major depression syndrome exhibited a phase-shifted profile of IL- 6 expression by $12 \mathrm{~h}$ compared to non-depressed healthy subjects. Circadian disruption is also occasionally seen in cancer patients. Among patients with different cancer diseases, disruption of the circadian rhythm has been noted in endocrine (e.g. cortisol and melatonin), metabolic (e.g. proteins and enzymes) and immunological components (e.g. cytokines) [41]. Whether the circadian dysregulation is a cause or a consequence of a stressful condition is a topic of ongoing research.

As was noted earlier in this review, disrupted biological rhythms are often associated with negative clinical outcomes. Studies in shift workers showed that night working is a risk factor in several types of cancers such as prostate, breast, endometrial, and colon cancers. Furthermore, other diseases such as obesity, diabetes, and cardiovascular disease seem to be more common among shift workers. Mormont et al. [42] showed that patients with poor circadian rhythmicity of sleep/activity cycles had a 5 -fold higher risk of dying within 2 years than the patients with a better circadian rhythmicity. As a result, there may be a relationship between circadian rhythmicity and disease development. This notion raises the pos- 
sibility that reinforcement of disrupted biological rhythms may reset the circadian clock and further improve outcome. Recently, there have been efforts particularly in cancer treatment in order to experimentally investigate the concept of biological rhythm reprogramming. This approach may have a transformative impact on clinical care, given that current standard practices proceed with little regard for circadian rhythms. Incessant lighting, continuous feeding, and other processes of care remove the external cues that normally maintain circadian patterns. If recovery from cancer or critical illness is indeed linked to the recovery of endogenous biological rhythms, optimizing the timing of care to re-engage key-immunoregulatory clock mechanisms could improve outcome for those patients. Preliminary results linking circadian rhythms and recovery are promising. $\mathrm{Li}$ et al. [43] showed that intermittent feeding for $2 \mathrm{~h} /$ day induces 24 -hour rhythmic expression of critical genes in clock-deficient tumors. Further, mice fed intermittently $2 \mathrm{~h} /$ day exhibit $\sim 40 \%$ less tumor growth than mice fed ad libitum emphasizing that meal timing cannot only enhance circadian rhythmicity but also improve survival. Similar results have been obtained for mice with Glasgow osteosarcoma, where restricted feeding induced an increase in the amplitude of temperature and activity diurnal rhythms [44]. Importantly, both of these studies indicated that intermittent feeding reinforced endogenous rhythmicity independent of its caloric composition, which further underlines the specific importance of feeding time. Along the same lines, Filipski and Levi [45] showed that altering feeding time can counterbalance the detrimental effect of chronic jet lag on tumor growth. In particular, restricted feeding prevented the circadian disruption originally induced by chronic jet lag in the liver and slowed cancer progression. Restricted feeding in arrhythmic (SCN-ablated) rats also restores rhythmicity of the pineal-gland melatonin secretion [46], highlighting the possibility that melatonin may also play a role in the resetting of biological rhythms in chronically stressed patients. In fact, oral melatonin administration has been found to restore sleep efficiency in tracheostomy patients who had lost circadian rhythmicity in endogenous melatonin [47]. In addition, Blask et al. [48] used a tumor perfusion model to show that rhythmic output of melatonin suppresses breast cancer proliferation in rats. Finally, melatonin regulation of cancer progression both in C57BL/6 null mice that exhibit lower levels of dark-phase melatonin and in B6D2f1 jet-lagged mice that exhibit atypical melatonin production mainly due to increased light phase production raises the possibility that rhythmicity of melatonin may play a more important role than its absolute concentration [49].

\section{Conclusions}

Homeostasis is maintained in the body through a large number of bidirectional interactions among physiological systems. The circadian and immune systems play leading roles in this dynamic behavior, and their effective regulation is of utmost importance. Experimental evidence shows that chronically stressful conditions often disturb the rhythmic orchestration of the body, resulting in blunted circadian rhythmicity of critical endocrine signals. Due to the highly complex, multilevel network of interactions between the circadian clock and inflammatory responses, a system-level approach is attractive in order to elucidate the underlying dynamics and design potential clinical interventions.

Significant progress along these lines has been made through studying mathematical models that elucidate the properties of circadian clocks relative to its entrainment dynamics and interactions with inflammatory mediators. These models illustrate the predictive power of mathematical approaches that also enable broader comprehensiveness of the biological system. In addition, their application in the context of the immune response based on current in silico studies seems promising. Further steps forward will continue to narrow the gap between scientific knowledge and clinical practice by incorporating systems biology approaches in real clinical intervention scenarios.

\section{Acknowledgments}

P.D.M. and I.P.A. acknowledge support from NIH(GM082974). P.D.M., J.D.S., and S.E.C. are supported, in part, by NIH GM34695.

\footnotetext{
References $\quad$ Castanon-Cervantes $\mathrm{O}, \mathrm{Wu}$ M, Ehlen JC, Paul K, Gamble KL, Johnson RL, Besing RC, Menaker M, Gewirtz AT, Davidson AJ: Dysregulation of inflammatory responses by chronic circadian disruption. J Immunol 2010;185:5796-5805.

- Bornstein SR, Licinio J, Tauchnitz R, Engelmann L, Negrao AB, Gold P, Chrousos GP: Plasma leptin levels are increased in survivors of acute sepsis: associated loss of diurnal rhythm, in cortisol and leptin secretion. J Clin Endocrinol Metab 1998;83:280-283.
} 
-3 Sephton SE, Sapolsky RM, Kraemer HC, Spiegel D: Diurnal cortisol rhythm as a predictor of breast cancer survival. J Natl Cancer Inst 2000;92:994-1000.

4 Leloup JC, Goldbeter A: Toward a detailed computational model for the mammalian circadian clock. Proc Natl Acad Sci USA 2003;100:7051-7056.

5 Mirsky HP, Liu AC, Welsh DK, Kay SA, Doyle FJ 3rd: A model of the cell-autonomous mammalian circadian clock. Proc Natl Acad Sci USA 2009;106:11107-11112.

6 Relogio A, Westermark PO, Wallach T, Schellenberg K, Kramer A, Herzel H: Tuning the mammalian circadian clock: robust synergy of two loops. PLoS Comput Biol 2011; 7:e1002309.

7 Becker-Weimann S, Wolf J, Herzel H, Kramer A: Modeling feedback loops of the mammalian circadian oscillator. Biophys J 2004; 87:3023-3034.

$\checkmark 8$ Gallego M, Eide EJ, Woolf MF, Virshup DM, Forger DB: An opposite role for tau in circadian rhythms revealed by mathematical modeling. Proc Natl Acad Sci USA 2006;103: 10618-10623.

-9 Westermark PO, Welsh DK, Okamura H, Herzel H: Quantification of circadian rhythms in single cells. PLoS Comput Biol 2009;5:e1000580.

-10 Nagoshi E, Saini C, Bauer C, Laroche T, Naef F, Schibler U: Circadian gene expression in individual fibroblasts: cell-autonomous and self-sustained oscillators pass time to daughter cells. Cell 2004;119:693-705.

-11 Mavroudis PD, Scheff JD, Calvano SE, Lowry SF, Androulakis IP: Entrainment of peripheral clock genes by cortisol. Physiol Genomics 2012;44:607-621.

-12 Abraham U, Granada AE, Westermark PO, Heine M, Kramer A, Herzel H: Coupling governs entrainment range of circadian clocks. Mol Syst Biol 2010;6:438.

-13 Antle MC, Foley DK, Foley NC, Silver R: Gates and oscillators: a network model of the brain clock. J Biol Rhythms 2003;18:339350.

-14 Antle MC, Foley NC, Foley DK, Silver R: Gates and oscillators II: zeitgebers and the network model of the brain clock. J Biol Rhythms 2007;22:14-25.

15 Geier F, Becker-Weimann S, Kramer A, Herzel H: Entrainment in a model of the mammalian circadian oscillator. J Biol Rhythms 2005;20:83-93.

16 Gonze D, Bernard S, Waltermann C, Kramer A, Herzel H: Spontaneous synchronization of coupled circadian oscillators. Biophys J 2005;89:120-129.

17 Bernard S, Gonze D, Cajavec B, Herzel H, Kramer A: Synchronization-induced rhythmicity of circadian oscillators in the suprachiasmatic nucleus. PLoS Comput Biol 2007; 3:e68.
8 Arjona A, Sarkar DK: Are circadian rhythms the code of hypothalamic-immune communication? Insights from natural killer cells. Neurochem Res 2008;33:708-718.

19 Calvano SE, Xiao W, Richards DR, Felciano RM, Baker HV, Cho RJ, Chen RO, Brownstein BH, Cobb JP, Tschoeke SK, Miller-Graziano C, Moldawer LL, Mindrinos MN, Davis RW, Tompkins RG, Lowry SF: A networkbased analysis of systemic inflammation in humans. Nature 2005;437:1032-1037.

20 Haimovich B, Calvano J, Haimovich AD, Calvano SE, Coyle SM, Lowry SF: In vivo endotoxin synchronizes and suppresses clock gene expression in human peripheral blood leukocytes. Crit Care Med 2010;38:751-758.

-21 Scheff JD, Calvano SE, Lowry SF, Androulakis IP: Modeling the influence of circadian rhythms on the acute inflammatory response. J Theor Biol 2010;264:1068-1076.

22 Balsalobre A, Brown SA, Marcacci L, Tronche F, Kellendonk C, Reichardt HM, Schutz G, Schibler U: Resetting of circadian time in peripheral tissues by glucocorticoid signaling. Science 2000;289:2344-2347.

-23 Burioka N, Takata M, Okano Y, Ohdo S, Fukuoka $\mathrm{Y}$, Miyata $\mathrm{M}$, Takane $\mathrm{H}$, Endo $\mathrm{M}$, Suyama H, Shimizu E: Dexamethasone influences human clock gene expression in bronchial epithelium and peripheral blood mononuclear cells in vitro. Chronobiol Int 2005;22:585-590.

24 Reiter RJ: The melatonin rhythm: both a clock and a calendar. Experientia 1993;49: 654-664.

25 Logan RW, Sarkar DK: Circadian nature of immune function. Mol Cell Endocrinol 2012;349:82-90.

26 Tracey KJ: The inflammatory reflex. Nature 2002;420:853-859.

27 Pollmacher T, Mullington J, Korth C, Schreiber W, Hermann D, Orth A, Galanos C, Holsboer F: Diurnal variations in the human host response to endotoxin. J Infect Dis 1996;174:1040-1045

28 Meyer-Hermann M, Figge MT, Straub RH: Mathematical modeling of the circadian rhythm of key neuroendocrine-immune system players in rheumatoid arthritis: a systems biology approach. Arthritis Rheum 2009;60:2585-2594.

29 Hrushesky WJ, Wood PA: Circadian time structure of septic shock: timing is everything. J Infect Dis 1997;175:1283-1284.

30 Scheff JD, Mavroudis PD, Calvano SE, Lowry SF, Androulakis IP: Modeling autonomic regulation of cardiac function and heart rate variability in human endotoxemia. Physiol Genomics 2011;43:951-964.

31 Nava F, Carta G, Haynes LW: Lipopolysaccharide increases arginine-vasopressin release from rat suprachiasmatic nucleus slice cultures. Neurosci Lett 2000;288:228-230.
32 Marpegan L, Leone MJ, Katz ME, Sobrero PM, Bekinstein TA, Golombek DA: Diurnal variation in endotoxin-induced mortality in mice: correlation with proinflammatory factors. Chronobiol Int 2009;26:1430-1442.

33 Okada K, Yano M, Doki Y, Azama T, Iwanaga $\mathrm{H}$, Miki H, Nakayama M, Miyata $\mathrm{H}$, Takiguchi S, Fujiwara Y, Yasuda T, Ishida N, Monden M: Injection of LPS causes transient suppression of biological clock genes in rats. J Surg Res 2008;145:5-12.

34 Kwak Y, Lundkvist GB, Brask J, Davidson A, Menaker M, Kristensson K, Block GD: Interferon-gamma alters electrical activity and clock gene expression in suprachiasmatic nucleus neurons. J Biol Rhythms 2008;23: 150-159.

35 Beynon AL, Coogan AN: Diurnal, age, and immune regulation of interleukin- $1 \beta$ and interleukin-1 type 1 receptor in the mouse suprachiasmatic nucleus. Chronobiol Int 2010; 27:1546-1563.

36 Koyanagi S, Ohdo S: Alteration of intrinsic biological rhythms during interferon treatment and its possible mechanism. Mol Pharmacol 2002;62:1393-1399.

37 Cavadini G, Petrzilka S, Kohler P, Jud C, Tobler I, Birchler T, Fontana A: TNF- $\alpha$ suppresses the expression of clock genes by interfering with E-box-mediated transcription. Proc Natl Acad Sci USA 2007;104: 12843-12848.

38 Chrousos GP, Gold PW: A healthy body in a healthy mind - and vice versa - the damaging power of 'uncontrollable' stress. J Clin Endocrinol Metab 1998;83:1842-1845.

-39 Mundigler G, Delle-Karth G, Koreny M, Zehetgruber M, Steindl-Munda P, Marktl W, Ferti L, Siostrzonek P: Impaired circadian rhythm of melatonin secretion in sedated critically ill patients with severe sepsis. Crit Care Med 2002;30:536-540.

40 Alesci S, Martinez PE, Kelkar S, Ilias I, Ronsaville DS, Listwak SJ, Ayala AR, Licinio J, Gold HK, Kling MA, Chrousos GP, Gold PW: Major depression is associated with significant diurnal elevations in plasma interleukin-6 levels, a shift of its circadian rhythm, and loss of physiological complexity in its secretion: clinical implications. J Clin Endocrinol Metab 2005;90:2522-2530.

41 Sephton S, Spiegel D: Circadian disruption in cancer: a neuroendocrine-immune pathway from stress to disease? Brain Behav Immun 2003;17:321-328.

42 Mormont MC, Waterhouse J, Bleuzen P, Giacchetti S, Jami A, Bogdan A, Lellouch J, Misset JL, Touitou Y, Levi F: Marked 24-h rest/activity rhythms are associated with better quality of life, better response, and longer survival in patients with metastatic colorectal cancer and good performance status. Clin Cancer Res 2000;6:3038-3045. 
43 Li XM, Delaunay F, Dulong S, Claustrat B, Zampera S, Fujii Y, Teboul M, Beau J, Levi F: Cancer inhibition through circadian reprogramming of tumor transcriptome with meal timing. Cancer Res 2010;70:33513360.

44 Wu MW, Li XM, Xian LJ, Levi F: Effects of meal timing on tumor progression in mice. Life Sci 2004;75:1181-1193.

45 Filipski E, Levi F: Circadian disruption in experimental cancer processes. Integr Cancer Ther 2009;8:298-302.
46 Feillet CA, Mendoza J, Pevet P, Challet E: Restricted feeding restores rhythmicity in the pineal gland of arrhythmic suprachiasmaticlesioned rats. Eur J Neurosci 2008;28:24512458.

47 Bourne RS, Mills GH, Minelli C: Melatonin therapy to improve nocturnal sleep in critically ill patients: encouraging results from a small randomised controlled trial. Crit Care 2008;12:R52.
8 Blask DE, Brainard GC, Dauchy RT, Hanifin JP, Davidson LK, Krause JA, Sauer LA, Rivera-Bermudez MA, Dubocovich ML, Jasser SA, Lynch DT, Rollag MD, Zalatan F: Melatonin-depleted blood from premenopausal women exposed to light at night stimulates growth of human breast cancer xenografts in nude rats. Cancer Res 2005;65:1117411184.

49 Greene MW: Circadian rhythms and tumor growth. Cancer Lett 2012;318:115-123. 